\title{
Etude synthétique du problème de la modélisation des écoulements partiellement cavitants
}

\author{
A. Rowe \\ Ingénieur de recherches au CNRS
}

\section{Introduction}

L'intérêt manifesté depuis de nombreuses années vis-àvis du problème de la modélisation des écoulements cavitants est dû manifestement à des implications techniques et économiques particulièrement fortes qui aujourd'hui encore jouent un rôle moteur important. Au cours de cet exposé on s'intéressera plus particulièrement au problème de la modélisation de la cavitation partielle qu'on rencontre dans de nombreuses machines.

On doit à Helmoltz et à KIRCHOFF d'avoir jeté les bases d'une modélisation qui à l'époque était censée représenter un sillage de nature monophasique s'étendant à l'infini. Avant la guerre de 40 , un grand nombre d'études furent réalisées, visant à établir des modèles de fermeture permettant de rendre compte de sillages finis [1]. En même temps, des théorèmes d'existence et d'unicité, ainsi que des études portant sur les conditions de détachement des lignes libres et leur géométrie furent établies par des théoriciens dont en France l'école de VILLAT [2] est particulièrement représentative. L'ensemble de ces travaux eut pour motivation première le besoin de rendre compte de l'existence d'une traînée pour des corps placés dans un écoulement à potentiel (paradoxe de d'AlemberT).

L'étude des écoulements cavitants ouvrit aux recherches précédentes un champ d'application particulièrement vaste. Cependant ce n'est qu'au début des années cinquante que sous l'impulsion donnée aux Etats-Unis par la marine les progrès vont s'accélérer. A cette époque, la supercavitation semblait offrir des perspecti- ves de développements techniques considérables dans le domaine des hélices marines et des hydroptères. Deux méthodes de calcul vont se développer parallèlement : la méthode non linéaire reprise par Wu, T. Y. T [3] selon la technique proposée plusieurs années auparavant par LEVI-CivitA [4] et la méthode des petites perturbations développée par Tulin, M. P. [5] successivement au premier et au deuxième ordre. Pour des raisons touchant aux conditions pratiques d'utilisation la supercavitation ne donna pas lieu à de nombreuses réalisations. L'expérience scientifique acquise se reporta alors naturellement dans le domaine de la cavitation partielle, le fonctionnement des hélices ou des pompes en régime partiellement cavitant étant en effet un problème d'intérêt non négligeable. Vers le début des années 1970 , le programme spatial américain apporta une contribution à ces études. L'exemple le plus achevé des calculs analytiques en théorie non linéaire a alors peut-être été dû à FURUYA qui en 1980 [7] traita le problème d'une grille d'aube partiellement cavitante, après avoir traité en 1975 [6] le problème d'une aile supercavitante tridimensionnelle placée sous une surface libre.

Les méthodes analytiques non linéaires dont les principes de mise en œuvre ont été mis en place par LEVICIVITA souffrent de faiblesses qui rendent aujourd'hui nécessaire l'utilisation des méthodes numériques. Ne disposant pas d'ordinateur, LEVI-CIVITA n'était capable que de résoudre le problème indéterminé. Le problème direct fut résolu par WU pour des profils de forme très simple tandis que FURUYA étendit "en principe" la méthode à des profils de forme quelconque grâce à

\section{Overview of the partially cavitating flows modelling problem.}

After a brief historical review, some of the difficulties directly linked to the problems of modelizing partially cavitating flows are analysed. Afterwards, an outline is given concerning the critical study of some of the models currently available in current literature, followed by the presentation of a model which was drawn up at the CREMHyG four years ago. The ability of the model to adapt to various ways of calculating (singular points or finite elements) and to account for the extremely various operational conditions is demonstrated via several models : such as the case of a single profile placed in a canal; an identical example with significant confinement effects and viscous effects; examples of two cavities being formed around an asymmetrical body whilst accounting for a gravitation effect : collar of centrifugal pump. 
l'utilisation devenue banale des ordinateurs. A noter qu'aujourd'hui les méthodes analytiques bien que conservant un indéniable intérêt didactique ne peuvent plus être considérées comme opérationnelles industriellement, la convergence des solutions ne pouvant être garantie.

Assez curieusement, au moment où l'avènement des méthodes numériques aurait permis d'ouvrir des perspectives de développement intéressantes, l'impulsion donnée aux Etats-Unis commença à s'essoufler. Ce furent le Japon, puis les européens qui prirent le relais, d'abord soutenus par les marines nationales. Au Japon, MrYAмото a joué le rôle d'un précurseur en traitant numériquement le problème d'une aile supercavitante suivant une formulation utilisant le potentiel d'accélération comme fonction inconnue [8]. Le cas qui nous intéresse de la cavitation partielle fut traité par NishIYAMA et ITO [9], YAMAGUCHI et KATO [10], puis à nouveau par ITO [11]. En France, il fallut attendre 1984 pour voir apparaître un premier modèle numérique non linéaire de cavitation partielle [12]. Entre temps, quelques études ponctuelles [13] et [14] avaient montré la possibilité de traiter le problème «Quasi-stationnaire ».

\section{Analyse du problème}

La cavitation partielle est gouvernée par des transferts interfaciaux de masse, de quantité de mouvement et d'énergie. Ces transferts sont eux-mêmes dépendants de la dynamique de l'écoulement de la phase liquide et de la dynamique de l'écoulement de la phase vapeur. Faute de savoir appréhender dans le détail ces divers mécanismes, la modélisation des écoulements partiellement cavitants s'est faite jusqu'à présent suivant des schémas assez sommaires dans lesquels seul l'écoulement liquide supposé potentiel est pris en compte.

\section{Hypothèses de base}

1. La géométrie de la cavité et la répartition des pressions sont calculées en supposant la pression constante dans la cavité et en négligeant le transfert de masse à l'interface. Ces hypothèses le plus souvent acceptables à la condition de prendre en compte la pression partielle des gaz dissous deviennent irréalistes lorsqu'il y a effet thermique. Cet effet se manifeste pour des fluides pour lesquels la chaleur latente de vaporisation est grande visà-vis de la chaleur spécifique du liquide. Dans un tel cas, l'énergie nécessaire à la vaporisation du fluide qui transite dans la cavité, prise à une mince sous-couche de liquide voisine de l'interface provoque le refroidissement de cette sous-couche. La température dans la cavité s'ajuste alors à une valeur $T_{\text {cav. }}<T_{\infty}$. Une pression s'établit correspondant en première approximation à l'équilibre thermodynamique. Un modèle prédictif ne peut alors ignorer les échanges entre la phase liquide et la phase vapeur [15].

2. S'en tenant dans le reste de cet exposé aux fluides non thermosensibles, une seconde hypothèse consiste à admettre que, dans l'incapacité où l'on est de rendre compte de l'aspect instationnaire vrai de l'écoulement, principalement dans la zone de fermeture des cavités, un écoulement potentiel stationnaire peut rendre compte de l'aspect moyenné de l'écoulement vrai. L'art du chercheur consistera à trouver un modèle de fermeture permettant d'assurer au mieux le bien-fondé de la démarche. Parmi les modèles existants, le modèle le plus simple à cavité fermée correspond à un schéma non dissipatif. La validité de ce modèle est limitée au cas où la présence de la cavité perturbe peu l'écoulement en aval (cavités de faible longueur). Lorsque le sillage de la cavité devient important, il est nécessaire de rendre compte de la dissipation due aux effets visqueux. La méthode universellement admise consiste à introduire une épaisseur de déplacement pour rendre compte des effets dus au sillage. Bien que cette démarche puisse donner des résultats utiles, l'écoulement type NavierStokes qui prédomine à l'arrière de la cavité n'est malheureusement pas formellement réductible à un schéma type «couplage écoulement potentiel — épaisseur de déplacement $»$.

\section{Validation d'un modèle}

La relation entre la longueur de cavité $\ell$ et le nombre de cavitation $\sigma=\left(P_{\infty}-P_{v}\right) / \frac{1}{2} \rho V_{\infty}^{2}$ permet de se rendre compte de la validité d'un modèle en première approximation. Malheureusement lorsqu'il s'agit d'un modèle à cavité ouverte, l'ouverture $\delta$ à l'arrière de la cavité devient souvent réglable, le nombre de cavitation $\sigma$ dépendant de $\delta$. Le modèle devient non prédictif, sauf à avoir trouvé empiriquement une loi $\delta(\ell)$ permettant d'obtenir la relation correcte $\ell(\sigma)$. La validité du modèle est alors corroborée par la comparaison des gradients de pression théoriques et expérimentaux s'établissant longitudinalement, dont principalement la valeur de la recompression. A noter que dans le cas d'un modèle fermé la recompression peut s'ajuster, mais dans une mesure assez faible [12] ; la loi $\ell(\sigma)$ dépend légèrement de cet ajustement. Remarquons enfin qu'il existe une incertitude sur le relevé de la longùeur moyenne, ce qui crée une difficulté d'interprétation lorsque la cavité pulse.

\section{Prévision du point de détachement}

La connaissance du point de détachement des cavités est également nécessaire pour la prévision correcte de la loi $\ell(\sigma)$. Heureusement dans ce domaine des progrès substantiels ont été faits ces dernières années [16] sur la base des études effectuées auparavant par ARAKERI [17]. II semble aujourd'hui établi que la cavité est toujours précédée d'un point de décollement laminaire de la couche limite. Le fait intéressant consiste à remarquer que le point de détachement se stabilise à une position pour laquelle la distance entre ce point et le point de décollement de la couche limite tend à s'annuler. Sur la base de cette constatation, des critères simples peuvent être mis en œuvre pour trouver la position du point de détachement. Cependant la position trouvée est sensible au choix du critère choisi, la condition limite : abscisse du point de détachement $=$ abscisse du point de séparation (critère de la première bissectrice) n'étant jamais 

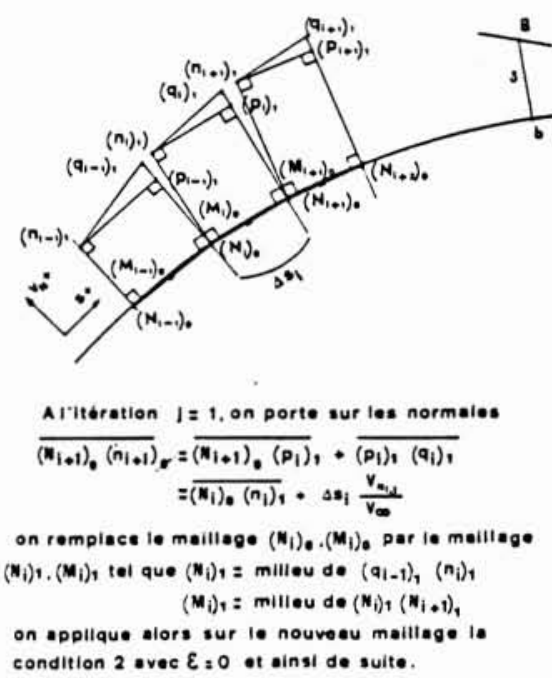

1. Relaxation de la géométrie d'une cavité.

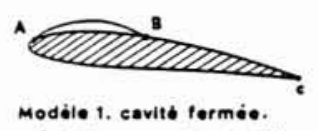

(schome non dissipatit)

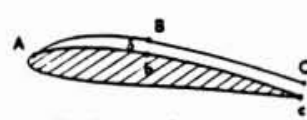

Moddle 2. cavité ouverte.

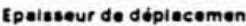

constante.

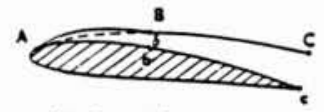

Modble 3. cavitb ouverte.

Epalsseur de dediecement

verient lineiairement.

ie s. Identique a modble 4. avec adjonction dun effet dotranspirntion le long de b c pour a juster la lol de recompreasion.

2. Représentation de quelques modèles numériques de fermeture.

rigoureusement réalisée. En écoulement axisymétrique, ARAKERI a proposé sur la base de plusieurs expériences, une relation empirique donnant l'écart entre les deux abscisses en fonction de paramètres caractérisant l'écoulement subcavitant au point de décollement laminaire (Epaisseur de quantité de mouvement de la couche limite, paramètre de Taylor-Saffman). Pour des profils bidimensionnels un critère équivalent n'a semble-t-il pas été établi, et les auteurs semblent utiliser le critère de la première bissectrice [10] avec un certain laxisme. En particulier, l'influence de la condition de sortie utilisée pour caractériser l'écoulement au point de détachement n'a pas été examinée alors que localement cette condition de sortie n'est pas toujours tangentielle. Dans le cas d'écoulements industriels à grand nombre de Reynolds, on manque d'informations sur la validité d'un critère lié au décollement laminaire de la couche limite.

\section{Aspect numérique}

Sous l'hypothèse d'un écoulement potentiel, deux techniques de calcul peuvent être utilisées: la technique des singularités dérivée d'une formulation intégrale et l'une des méthodes différences ou éléments finis. Chaque technique présente des avantages et des inconvénients propres bien répertoriés. On doit malheureusement faire remarquer qu'alors que la plupart des modèles à ce jour proposés font appel à la technique des singularités, les auteurs ne semblent guère se soucier, ni du débit de fuite associé à la discrétisation [10], ni de la dégénérescence du modèle lorsque les conditions deviennent subcavitantes [11], ni des oscillations qui peuvent apparaittre dans les zones de raccord cavité-profil, ni de l'incompatibilité qu'il y a entre le fait d'imposer une vitesse constante sur la cavité, et la condition de vitesse nulle au point d'arrêt lorsqu'il s'agit d'un modèle fermé « classique ». Pourtant, les aérodynamiciens se préocupent depuis longtemps du problème du débit de fuite; la dégénérescence du système de résolution matriciel lorsque les conditions sont subcavitantes est en général la marque d'un mauvais conditionnement en conditions cavitantes; enfin l'approximation faite généralement sur la condition de fermeture empêche une discrétisation propre à un traitement type éléments ou différences finies.

La dernière approximation mentionnée peut être levée en adjoignant à l'arrière de la cavité une zone de transition $B C$ (modèles $1^{\prime}$ à $4^{\prime}$ de la figure 2 ) permettant à la vitesse d'évoluer de manière non discontinue. Une méthode simple [12] consiste à raccorder la valeur de la vitesse normale sur la cavité, qui est non nulle avant la convergence de la géométrie, à une valeur nulle sur le corps. On est alors amené à écrire dans la zone de transition une condition limite du type :

$$
V_{N}=V_{N}\left\{\begin{array}{l}
\text { exprimée à l'arrière de la cavité } \\
\text { en fonction des inconnues }
\end{array}\right\} \cdot f(s)
$$

$$
f(s)=\left[\frac{s-s_{2}}{s_{1}-s_{2}}\right]^{\alpha}\left\{\begin{array}{c}
s=\text { abscisse d'un point courant } \\
s_{2}=\text { abscisse du point arrière } \\
\text { de la zone de transition } \\
s_{1}=\text { abscisse du point arrière } \\
\text { de la cavité }
\end{array}\right.
$$

$$
V_{N}=\text { Vitesse normale avant convergence }
$$

A la convergence, la vitesse normale étant nulle sur la cavité, la condition (1) assure bien $V_{N}=0$ sur tout le contour. La condition de fermeture discrétisée s'écrira préférentiellement :

$$
\sum_{i} \Delta s_{i} \frac{V_{N_{i, j}}}{V_{t_{i, j-1}}}=\varepsilon \delta
$$

où $\varepsilon=1$ à la première itération $j$, et $\varepsilon=0$ aux itérations suivantes. A la condition (2), on peut associer un schéma de construction géométrique permettant d'obtenir exactement la valeur $\delta$ du jeu à l'arrière de la cavité (figs. 1 et 2). 


\section{Critères de validité d'un modèle}

Les critères suivants devront être satisfaits :

1. Le modèle ne devra pas devenir inopérant lorsque les conditions deviennent subcavitantes.

2. Le débit de fuite associé à la discrétisation devra être minimisé (méthodes intégrales). Cette condition prend d'autant plus d'importance que les structures sont minces.

3. La description des zones de transition devra être assurée sans instabilités.

4. Un critère de convergence numérique facilitant les calculs devra être établi permettant de prévoir la position du point de détachement, basé sur la notion de décollement laminaire.

5. Le modèle devra prévoir correctement la loi $\ell(\sigma)$.

6. La forme de la recompression derrière la cavité devra être prévue correctement pour un domaine d'utilisation donné.

Comme il a été dit précédemment, la plupart des modèles existants dans la littérature ne répondent pas à la deuxième condition. Le modèle de ITO ne répond pas non plus à la première. Pour assurer la troisième condition, cet auteur a utilisé sur la cavité une répartition de sources de forme prédéterminée suivant un développement de Fourier du type :

$$
m=V_{\infty}\left[a_{0} \operatorname{tg} \frac{\tau}{2}+\sum_{n=1}^{N} a_{n} \sin n \tau\right]
$$

valable pour une condition de sortie tangentielle, le paramètre $\tau$ étant lié à l'abscisse par la relation :

$$
s=s_{0}+\frac{s_{1}-s_{0}}{2}[1-\cos \tau]
$$

$s_{0}=$ abscisse du point de détachement de la cavité.

La restitution exacte de la loi $\ell(\sigma)$ ne peut être assurée en toute rigueur que par un modèle ouvert. Cependant dans certains cas où l'effet dissipatif est faible, un modèle fermé peut donner une bonne approximation. Le manque d'un critère numérique précis répondant à la condition 4 induit une erreur en général du second ordre vis-àvis de l'erreur associée au choix du modèle de fermeture. De toutes les conditions précitées seule la condition 6 semble encore difficile à satisfaire encore que la condition 5 [loi $\ell(\sigma)]$ ne puisse être satisfaite sans référence préalable à l'expérience.

\section{Présentation de quelques modèles}

On a représenté sur la figure 2 des schémas correspondant à des modèles numériques de plus en plus complexes. Le modèle 1 non dissipatif qui suppose que la cavité se referme sur le corps a la particularité de surévaluer la valeur du $\sigma$ en donnant à l'écoulement une déviation trop grande. Hormis la difficulté précédemment mentionnée de la discontinuité en $B$, ce modèle restitue une loi de correspondance $\ell(\sigma)$ non biunivoque.

Le modèle 2 a été proposé par KATO [10], mais avec un schéma numérique sommaire dont les degrés de liberté ne sont pas en nombre suffisant pour régler la valeur de $\delta$. Ce modèle donne souvent des résultats meilleurs que ceux obtenus avec le modèle 1 , encore que le schéma numérique utilisé par KATO ne réponde pas à la deuxième condition de validité du paragraphe précédent (tourbillons d'intensité constante).

L'intérêt des modèles $2^{\prime}$ et $3^{\prime}$ a été montré en [18] pour des cas où les effets visqueux deviennent prépondérants. Le modèle 4 peut être mis en œuvre avec des singularités réparties soit sur la cavité, soit sur la paroi solide $A b$, soit à la fois sur la cavité et sur $A b$, si on utilise la technique MPPM (Minimisation Process Panel Method) présentée de manière extensive en [12]. Dans ce dernier cas, on impose une condition de vitesse constante sur la cavité, du côté de l'écoulement externe, et une condition d'imperméabilité sur $A b$. Le vecteur à minimiser, distribué le long du contour $A b c A B$ s'écrit :

$$
\begin{aligned}
& \left(q_{1}-q_{2}\right),\left(q_{2}-q_{3}\right), \ldots\left(q_{n-1}-q_{n}\right), \\
& \quad\left(\gamma_{1}-\gamma_{2}\right),\left(\gamma_{2}-\gamma_{3}\right), \ldots\left(\gamma_{n-1}-\gamma_{n}\right)
\end{aligned}
$$

où les $q_{i}$ et les $\gamma_{i}$ représentent les intensités des sources et des tourbillons. La minimisation impose un écoulement lisse à l'intérieur de la cavité malgré les fortes interactions dues à la proximité des frontières. Le caractère ouvert des modèles 4 et 5 pose le problème formel de l'unicité de la solution et de la réversibilité vis-à-vis de la loi $\delta(\ell)$ en raison du caractère itératif de la résolution. La validité de la lecture des coefficients de pression en aval de la cavité doit aussi être discutée dans le cas du modèle 4 [19]. Une application du modèle 5 est présentée ciaprès.

\section{Présentation de quelques résultats}

On présente ici quelques résultats obtenus à partir des modèles $1^{\prime}, 2,4$ et 5 mis en œuvre suivant un schéma numérique faisant appel soit à la technique MPPM soit à la technique des éléments finis. La figure 3 montre une comparaison entre les modèles $1^{\prime}$ et 2 à partir des résultats expérimentaux obtenus par KATO. La technique de calcul utilisée, dans les deux cas de type MPPM, permet de satisfaire aux conditions 1,2 et 3 du paragraphe 3. Le fait d'introduire un sillage proche dans le modèle 1 ne change pas sensiblement la valeur du $\sigma$ (voir [12]). Le comportement du modèle fermé est confirmé : on remarque que pour deux valeurs de la longueur de cavité on peut obtenir une même valeur du nombre de cavitation. A noter que la minimisation permet d'utiliser le modèle 2 soit en réglant à volonté le paramètre $\delta$, soit de manière prédictive le paramètre $\delta$ étant calculé à partir du critère de minimisation, ce qui a été réalisé dans le cas présent. Le modèle avec sillage d'épaisseur constante donne des résultats qui respectent mieux la pente $\mathrm{d} \ell / \mathrm{d} \sigma$, surtout pour les cavités longues.

Les figures 4 et 5 représentent deux résultats obtenus avec le modèle 4 dans le cas d'une géométrie confinée où les effets visqueux sont importants [18]. Le calcul est non prédictif en ce sens que l'ouverture est calculée à partir de la valeur expérimentale du $\sigma$ pris comme donnée d'entrée. Le caractère lisse de l'écoulement interne permet de donner à cet écoulement une signification 


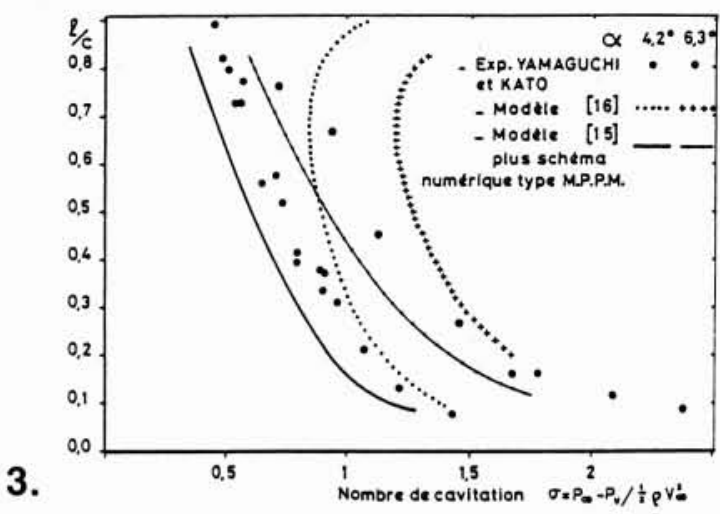

4.

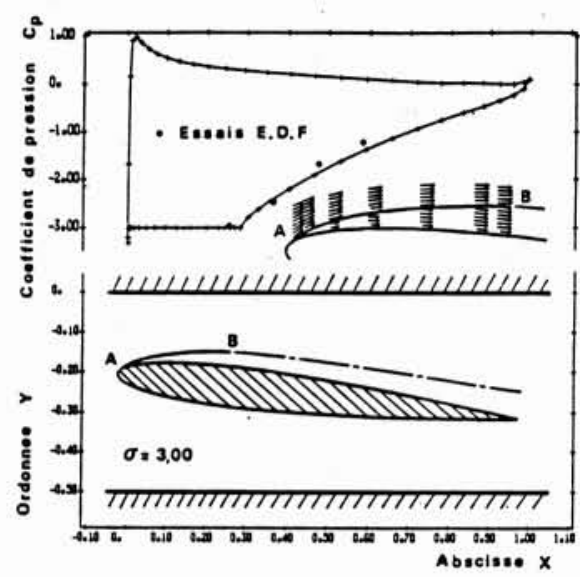

5.

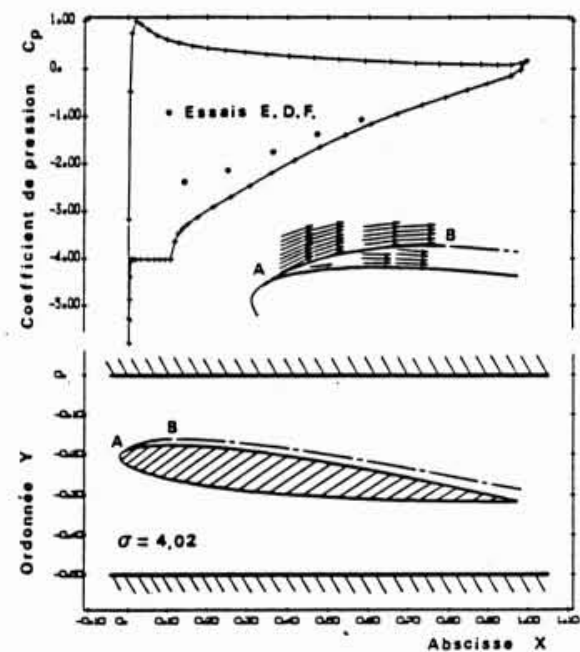

1. Corps exisymbtrique : condition de gllasement. 2. Cevite t pression constante.

3one de fermeture i beisseur de deplecement.

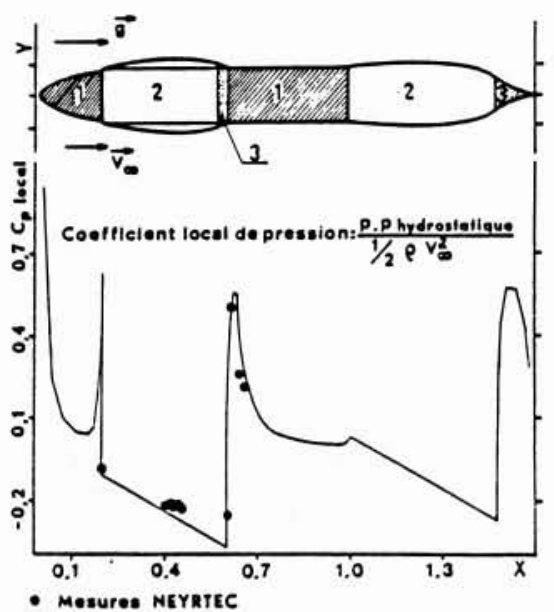

3. Comparaison de deux modèles simples avec les résultats expérimentaux de KaTo.

4. Effet de sillage dans un écoulement confiné. Comparaison des taux de recompression $(\ell / c=0.28)$.

5. Effet de sillage dans un écoulement confiné. Comparaison des taux de recompression $(\ell / c=0,10)$.

6. Calage du taux de recompression par effet de transpiration dans le sillage $(\ell / c=0,1 ; \sigma=4,02)$.

7. Calcul simultané de 2 cavités sur un corps axisymétrique à arrière tranqué en fluide pesant.

physique assez proche de la réalité : il y a vaporisation sur la cavité et glissement sur la partie $A b$ du profil. Malgré ce caractère lisse, on ne peut accorder au champ obtenu une valeur quantitative. Il y a en effet incompatibilité entre l'hypothèse d'une pression constante à l'intérieur de la cavité et la représentation potentielle utilisée, à supposer que les effets visqueux soient négligeables. Dans l'exemple présenté, le respect du critère 4 étant assuré automatiquement par le calcul, l'examen de la loi de recompression a seul un intérêt vis-à-vis de la validité du modèle. On constate que dans un cas la recompression est correctement décrite, alors que dans l'autre cas il y a discordance. L'adjonction d'un effet de transpiration derrière la cavité permet d'ajuster cette recompression (modèle 5, fig. 6), mais le modèle devient non prédictif à deux titres différents. Le dernier résultat montre la difficulté qu'il y a à rendre compte d'écoulements où les effets visqueux sont importants à partir d'un schéma potentiel.

Comme il a été dit précédemment, l'introduction d'un sillage proche donne au calcul un caractère rigoureux qui permet d'envisager le traitement de configurations complexes. A titre d'exemple, on présente figure 7 le cas d'un écoulement axisymétrique avec prise en compte simultanée de deux cavités en présence d'un effet de gravité. La relaxation de la cavité arrière demande des précautions particulières pour assurer la convergence.

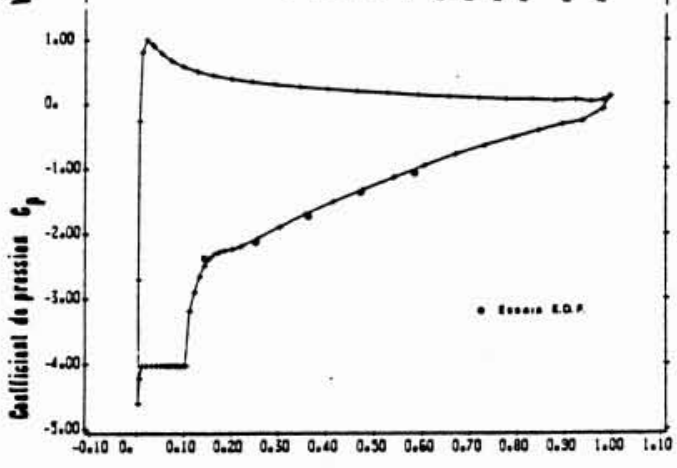




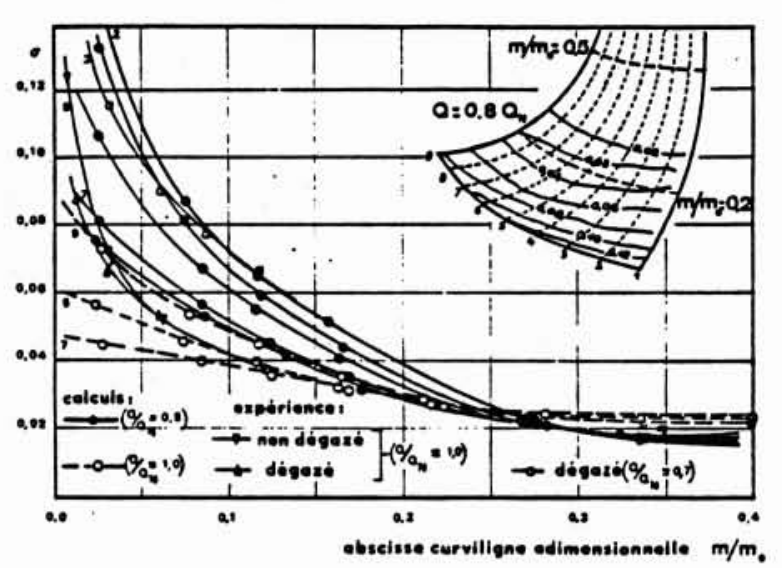

8. Longueur de la cavité en fonction du paramètre de cavitation $\sigma$ sur différentes surfaces de courant pour le rouet d'une pompe centrifuge (modèle SHF) au débit nominal et au débit partiel 0,8 Qn.

La condition de pression constante dans la cavité se traduit en effet par une condition de vitesse tangente non linéaire vis-à-vis de la vitesse au point de détachement :

$$
\left\{\begin{aligned}
\vec{V} \cdot \vec{t} & =V_{D}\left[1+\frac{2}{F} \cdot \frac{x-L}{L} \cdot \frac{V_{\infty}^{2}}{V_{D}^{2}}\right]^{\frac{1}{2}} \\
F^{2} & =V_{\infty}^{2} / 2 g L
\end{aligned}\right.
$$

où $V_{D}$ est la vitesse au point de détachement et $F$ le nombre de Froude basé sur la longueur $L$ du corps. La condition de fermeture doit être ajustée progressivement, et pour chaque position intermédiaire du point arrière de la cavité, la fonction (6) doit être calculée itérativement [12]. Le modèle de fermeture utilisé, de type $1^{\prime}$, s'accommode bien d'une géométrie tendue; on constate que grâce à l'ajustement du sillage proche effectué dans un domaine d'étendue non négligeable [20] on obtient à la fois une valeur correcte de $\sigma$ et une valeur correcte du taux de recompression. A noter aussi le gonflement de la cavité de culot dû à l'effet de gravité.

La figure 8 est une illustration de l'utilisation du même modèle $1^{\prime}$ appliqué au calcul aube à aube d'une pompe centrifuge suivant la technique des éléments finis, dans le cadre d'une méthode type S1/S2 [21]. Pour chaque pompe élémentaire de l'écoulement méridien, la cavité est calculée d'une manière quasi-bidimensionnelle, la vitesse relative sur la cavité au point d'accrochage $W_{A}$ étant prise comme inconnue. La condition de pressions constante dans la cavité permet d'exprimer en tout point de la cavité la vitesse locale $W_{M}$ en fonction de $W_{A}$ de la manière suivante :

$$
W_{M}=W_{A}\left(1+\omega^{2} \frac{r_{M}^{2}-r_{A}^{2}}{W_{A}^{2}}\right)^{\frac{1}{2}}
$$

La condition de fermeture (2) peut s'appliquer à la condition de remplacer en chaque point la vitesse tangente par l'expression (7) dans l'équivalent discrétisé de (2). La discrétisation faisant intervenir les valeurs de la fonction de courant à chaque nœud du maillage, la condition discrétisée (2) devient :

$$
\begin{aligned}
\frac{\psi_{B}}{b_{B} W_{B}}+\sum_{i} \psi_{i}\left(\frac{1}{b_{i-1}\left(W_{M}\right)_{i-1}}-\frac{1}{b_{i}\left(W_{M}\right)_{i}}\right) & = \\
& =\frac{\psi_{A}}{b_{A} W_{A}}
\end{aligned}
$$

$b=$ paramètre géométrique connu.

La construction, rigoureuse en fin de convergence, revient à admettre qu'on peut, en début d'itération assimiler la vitesse tangente à la vitesse relative. L'analyse de la figure 8 permet de retrouver le comportement des poches observé dans un rouet de pompe : blocage dû à l'effet de recouvrement, aspect tridimensionnel des poches, influence du débit.

\section{Conclusion}

Les techniques numériques élaborées ces dernières années peuvent s'adapter sans difficulté au traitement des écoulements partiellement cavitants dans les situations les plus complexes. L'introduction de conditions aux limites variant progressivement dans le sillage, justifiée par la notion d'épaisseur de déplacement, pour l'écoulement fictif moyenné, conduit à une formulation s'adaptant aussi bien aux méthodes intégrales qu'au traitement direct des équations différentielles. Les méthodes intégrales demandent cependant de prendre certaines précautions pour minimiser le débit de fuite associé à la discrétisation.

L'utilisation des ordinateurs performants dont on dispose aujourd'hui rend possible le calcul de structures tridimensionnelles complexes tels que pompes hélices, clapets, etc... Un effort important reste à faire pour assurer le calage des modèles pour des conditions de fonctionnement industrielles déterminées notamment la prévision de la recompression.

Adresse de l'auteur

Monsieur A. Rowe.

Centre de recherches et d'essais de machines hydrauliques de Grenoble (CREMHy G)

ENSHMG - BP 95

38402 Saint-Martin d'Hères Cedex

Tél. : 1676448263

où $r$ est la valeur du rayon local, et $\omega$ la vitesse angulaire de rotation. 


\section{Bibliographie}

[1] Brillouin: “Annales de Physique et de Chimie », t. 23, p. $154,1911$.

[2] VILLAT: "Sur la résistance des fluides " Thèse ; Annales de l'Ecole Normale Supérieure, 1911. Voir aussi le livre de VILLAT « Aperçus théoriques sur la résistance des fluides", Collection Scientia Gauthier-Villars, 1920.

[3] WU T. Y. T. : «A wake model for free streamline flow theory, Part 1: Fully and partially developed wake flows and cavity flows past an oblique flat plate », J. Fluid. Mech., 13, pp. 161-181, June 1962.

[4] Levi-Civita : «Scie le leggi di resistenge », Rendiconti del Circolo Mathematics di Palermo, t. 23, 1907.

[5] TULIN M. P. : «Supercavitating flows, small perturbation theory ", Journal of Ship Research, Vol. 7, pp. 16-37, 1964.

[6] Furuya O.: " Three dimensional theory on supercavitating hydrofoils near a free surface ", J. Fluid Mech., 71, pp. 339-359, 1975.

[7] FURUYA O.: « Non linear theory for partially cavitating cascade flows ", IAHR 10th Symposium. Tokyo, pp. 221241,1980 .

[8] Mічамото M. : « Characteristics of supercavitating hydrofoils with finite span ", Trans. JSME, vol. 36, $\mathrm{n}^{\circ} 289$, pp. 1485-1492, 1970.

[9] Nishiyama T., Ito J. : « Calculation of partially cavitating flow by singularity method (part 1, two dimensional isolated hydrofoil) », Trans. JSME, vol. 43, n` 370 , pp. 2165 2174, July 1977.

[10] Yamaguchi H., Kato H. : "Non linear theory for partially cavitating hydrofoils ", Trans JSNA, vol. 152, pp. 117-124, Jan. 1983.

[11] Iто J. : « Calculation of partially cavitating thick hydrofoil and examination of a flow model at cavity termination ", International Symposium on Cavitation. Sendaï, Japan, April 1986.
[12] Lemonnier H. and Rowe A. : " Another approach in modelling cavitating flows ", J. Fluid Mech. A paraître.

[13] Furness R. A., and Hutton S. P. : « Experimental and theoritical studies of two-dimensionnal fixed-type cavitics », J. of Fluids Engineering. Déc. 1975, pp. 515-522.

[14] Chang-Sup L. : « Prediction of the transient cavitation on marine propellers by numerical lifting-surface theory ", Thirteenth Symposium on Naval Hydrodynamic, vol. 1, Session 1, Tokyo, oct. 1980.

[15] Kato H. : « Thermodynamic effect on incipient and developed sheet cavitation ", International Symposium on Cavitation Inception 1984. Edit; B. R. PARKIN et W. B. MORGAN.

[16] Franc J. P. and Michel J. M. : * Attached cavitation and the boundary layer : experimental investigation and numerical treatment », J. Fluid Mechanics, vol. 154, pp. 63-90, 1985 .

[17] ARakerI V. H. : *Viscous effect on the position of cavitation separation from smooth bodies ", J. Fluid Mechanics, vol. 68-4, pp. 779-799, 1975.

[18] Pellone C. and Rowe A. : « Effect of separation on partial cavitation ", J. of Fluids Engineering, vol. 110, $\mathrm{n}^{\circ} 2$, pp. 182-189, 1988.

[19] Blottiaux O. and Rowe A. : «Aspects of partially cavitating flow modelling , à paraitre.

[20] Reboud J. L., Rowe A. et Lemonnier H. : « Etudes des écoulements cavitants autour de corps axisymétriques à arrière tronqué, avec prise en compte d'un effet de gravité ATMA, Session 1985.

[21] Schultz J. L. : "Modélisation de la cavitation partielle dans les pompes ", thèse, CREMHyG, janvier 1988.

Ce travail représente une synthèse de travaux réalisés avec l'aide de différents organismes dont principalement le CNES, EDF, l'Aérospatiale et la SEP. 
M. AVELLAN : fait remarquer que les conditions de bord de fuite jouent un rôle fondamental et que les pressions en régimes cavitant et non cavitant doivent être les mêmes au bord de fuite c'est d'ailleurs ce qui a été observé expérimentalement. Par ailleurs, M. AvelLAN se demande si on ne devrait pas faire un calcul visqueux?

$M$. RoWE : rappelle que ses travaux ont été réalisés avec peu de résultats expérimentaux et que les calculs qui ont été faits sur la géométrie essayée à EDF correspondent à des conditions sévères dues principalement à un confinement très important et à une grande incidence. Cela le conduit à être plus optimiste pour des cas industriels généralement plus simples. M. RowE pense qu'un calcul visqueux n'est pas réaliste à l'heure actuelle du fait principalement que la turbulence due à la cavité est d'un type très particulier dans la mesure où elle est conditionnée par l'aspect diphasique de l'écoulement.

M. DESCLAUX: pense qu'il y a probablement un choix à faire entre le modèle à cavité fermée et le modèle à sillage et que la réponse n'est certainement pas universelle; le modèle à cavité fermée lui apparaît mieux adapté au cas des petites poches, alors que celui à sillage lui apparaît mieux adapté au cas d'une cavité plus développée.

M. VERRY: pense que le modèle ouvert est difficile à mettre en auvre pour une pompe centrifuge et qu'il faut s'en tenir au modèle fermé. Sur le problème de l'équilibre transversal méridien, M. VERRY fait remarquer que dans le cas de la pompe SHF, la poche était bien répartie du plafond à la ceinture et pourtant le calcul donnait des poches plus longues au plafond qu'à la ceinture

M. ROWE: dit que cela peut être attribué au problème du couplage entre calcul méridien et calcul aube à aube.

M. VERRY: pense que l'on peut distinguer typiquement deux régimes : le régime des grandes incidences, et le régime des petites incidences, et qu'il faut probablement faire appel à deux modèles différents.

M. ROWE : En réponse à une question précédente de M. VERRY, se demande pourquoi on ne pourrait pas utiliser de modèle ouvert en pompe centrifuge.

M. DESCLAUX : Pour compléter ce qu'a dit M. VERRY... j'ajoute qu'en ce qui concerne l'équilibre radial il faudrait déjà faire la part des choses entre ce qu'on a expérimentalement et ce que donne un calcul potentiel non cavitant, avant d'accuser le calcul cavitant.

Cela n'a pas été fait, alors on a du mal à porter un jugement là-dessus.

Par ailleurs, en ce qui concerne les modèles avec sillage ouvert, il ne faut pas oublier que même dans une pompe centrifuge il arrive un moment où la performance se dégrade. Il n'est pas évident que ce soit toujours un modèle à cavité fermée qui se rapproche de la situation des poches de cavitation.

M. le Président: Mais le problème de modélisation de la cavitation porte en lui-même une difficulté : c'est qu'on calcule une poche, et que de toute manière le problème ressemble à ce qu'on appelle en aérodynamique "un calcul inverse", un calcul de forme. Par conséquent on dispose d'un grand nombre de degrés de liberté et on joue sur toutes sortes de conditions plus ou moins bien fondées; à l'issue du calcul on fait correspondre une loi de coefficient de pression à une forme de profil.

A-t-on alors modélisé la cavitation? La présence d'une poche se traduit-elle réellement par une modification des coefficients d'efforts?

Il me semble que c'est la difficulté fondamentale de la modélisation des poches de cavitation, qu'on rencontre même en écoulement bidimensionnel.

M. ROWE : Je suis d'accord avec cette analyse ; mais la difficulté est d'autant plus grave que ces pertes sont liées à ce qui se passe à l'arrière de la cavité... et que cela, on sait très mal l'appréhender.

M. AVELLAN : demande à M. Rowe quels types de singularités sont utilisées dans le modèle de cavité ouverte et se demande si des instabilités de type Kelvin Helmotz ne peuvent pas se produire dans le cas d'une nappe tourbillonnaire.

$M$. ROWE: répond qu'il s'agit de sources et tourbillons et que, bien que quelques précautions soient à prendre dans le cas d'une ligne de singularités continue, c'est surtout dans le cas de la méthode des vortex discrets que le caractère individualisé des tourbillons peut soulever ce type de difficultés.

M. AVELLAN : fait mention des travaux de modélisation qui sont menés à l'IMHEF dans ce domaine et qui s'apparentent plutôt à un calcul de type inverse. Il affirme que de bons résultats ont été obtenus en particulier en choisissant une longueur de sillage la plus courte possible et en imposant des pressions au bord de fuite identiques à celles du régime subcavitant.

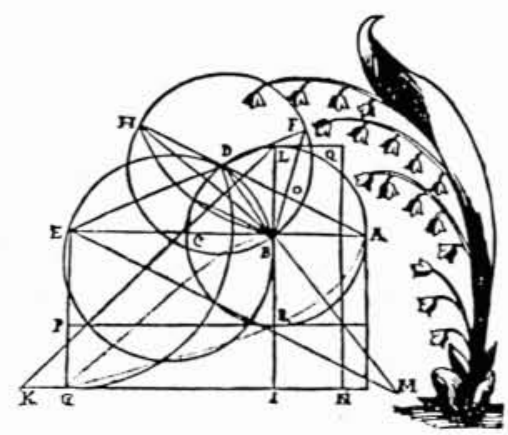

